\title{
42. CLOSE BINARY STARS (ÉTOILES DOUBLES SERRÉES)
}

\section{PRESIDENT:}

VICE-PRESIDENT:

ORGANIZING COMMITTEE:

J. V. Clausen, H. Drechsel, P. P. Eggleton, B. J. Geldzahler, G. Hill, S. J. Kenyon, P. Szkody, R. E. Taam, A. V. Tutukov, O. Vilhu, J. C. Wheeler, A. Yamasaki, Di-S. Zhai

\section{INTRODUCTION}

The pervasive connection of Close Binary Stars (CBS) with essentially every fundamental field of astronomy and astrophysics has required a huge number of papers on many topics to be considered for this report. As a consequence, most of the invited contributors were forced to use more space then alloted. This coupled with the tight contraints imposed by the crckapb style did require the Commission's President to make possibly controversial and certainly painful choices on how and where to make some unavoidable cuts. In particular, I had to drop most of the authors' names in the references throught the text. This much suffered but necessary decision resulted in a $20 \%$ reduction of the report length. I hope that only a minority of $\mathrm{C} 42$ members will not share my choice. Since the various contributions were already quite concise, the remaining alternative was that of dropping some of the science, a choice I did actually consider, I must confess, only briefly.

The excellent status of CBS research is demonstrated by the number and quality of conference proceedings and specialized books published in the past triennium. Outstanding reviews on various aspects of CBS research can be found in New Frontiers in Binary Star Research (APS Conf Ser 38), The Impact of Long Term Monitoring of Variable Star Research (NATOASI Wrks, Kluwer), Interacting Binaries (ASP Conf Ser 56), The Origins, Evolution, and Destinies of Binary Stars in Clusters (ASP Conf Ser 90), 22nd Saas-Fee Advanced Course in Interacting Binaries (Springer-Verlag), The Realm of Interacting Binary Stars (Ap\&SSci Library 177), Cataclismic Variables (Ap\&SSci Library 205), Cataclysmic Variable Stars (Cambridge 
Univ Press, Ap Ser 28), Accretion Disks in Compact Stellar Systems (Adv Ser Ap \& Cosmol 9, World Sci), Accretion Phenomena and Related Outflows (Kluwer), X-ray Binaries (Cambridge Univ Press, Ap Ser 26), Compact Stars in Binaries (IAU Symp 165), Stellar Jetsand Outflows (Kluwer).

As an introduction to the specific topics beautifully presented in the following sections by competent experts, I should like to mention: on the observational side, the discovery of the microlensing phenomenon; the polarisation measurements of compact objects; the now mature field of systematic, multi-band, long-term monitoring programmes to penetrate the real behaviour and physics of active CBS; the full exploitation of the spectacular imaging and spectroscopic capability of HST for stellar work, that will be hopefully praised in the coming triennium with a more generous than presently allocated HST time; and, on the interpretation and theoretical side, the implementation of more sophysticated computer codes to map starspots, plages, magnetic fields, and mass flows; the continued improvement of light curve modeling; the advanced modeling of wind streams, accretion disks, and CBS formation, as a result of fragmentation.

On the organisation side, it should be mentioned: a) the continued and invaluable service offered by the Bibliography and Program Notes on $C B S$, now under a renewed editorial board and Editor-in-chief, Horst Drechsel, who took over benefiting of the outstanding work done by his predecessor Atsuma Yamasaki, b) the reshape, not simply from the cosmetics point of view and easy accessibility through WWW (http://www.konkoly.hu/IBVS $/ I B V S . h t m l)$, thanks to the dedicated editors at Konkoly Observatory and Chairman Chris Sterken. Since the previous triennium, IBVS is being jointly sponsored by Commissions $27 \& 42$, now adhering to the new IAU Division $V$ (Variable Stars), with Yoji Kondo as President.

Finally, I should like to render a due, but sincere tribute to one of our best friend, the long-lasting and fantastic satellite International Ultraviolet Explore (IUE) while is being switched off just in these days, after an astonishing performance lasting close to two decades and having accompanied more than one generation of astronomers in their scientific growth, particularly in the field of active CBS. A modern and more powerful replica of IUE is very much desirable and necessary. In our field, the outstanding contribution of the most recent X-ray, EUV and IR satellites, notably ROSAT, GINGA, EUVE, and IRAS should at least be mentioned here, anticipating their many quotations in this report. Nor I can leave unmentioned the presently operating and future satellites, such as SAX, ISO, XMM, FUSE, and the series of SPECTRUM missions. It is an easy task to anticipate their tantamount relevance to CBS studies in years to come. 


\section{STATISTICAL INFORMATION (Horst Drechsel)}

Data banks. The Internet provides the basis for worldwide computer networking and data exchange. An enormous number of data banks and archives are accessible via $W W W$ or $f t p$, which are of interest for CBS researchers. Only a few well established sites, as entry points for further links, are here mentioned.

The Centre de Données Astronomique de Strasbourg, CDS (http://cds arc.u-strasbg.fr), maintains the SIMBAD astronomical database, which comprises data for about 1 million objects and offers access to the AstroWeb, a collection of pointers to astronomically relevant Internet resources.

The ESO/STECF Archive (http://arch-http.hq.eso.org) is a collaboration of ESO, ST-ECF, and the Canadian Astronomy Data Centre (CADC) at the Herzberg Institute of Astrophysics. One can query a large number of data bases using STARCAT, a user interface to a set of services available to astronomers and archival researchers. The archive contains important ESO and HST data bases, all major astronomical catalogues, the Digital Sky Survey (DSS) and it HST Guide Star Catalogue (GSC).

The CADC (http://cadcwww.dao.nrc.ca) provides links to the HST, CFHT, JCMT and other archives, the GSC catalogue, the DSS, and to SIMBAD.

Catalogues and atlas. During the period 1993-1996 several new catalogues containing data on CBS have been published.

The Long-Term Photometry of Variables (LTPV) program at ESO was continued with Strömgren observations from 1986 through 1994. The data were published as $2 n d, 3 r d$, and 4 th data catalogues (A\&AS 102, 79; 109, $329 ; 113,31$, and are also available in electronic form from the CDS.

As a by-product of the Optical Gravitational Lensing Experiment (OGLE), a total of 581 new eclipsing binaries were found in the Galactic bulge, and are listed in two catalogues (ActaA 44, 317; 45, 1). Similarly, another important achievement was the identification of 79 new eclipsing binaries in the bar of the LMC in the course of the French EROS microlensing project, which actually doubled the number of known eclipsing binaries in the LMC (A\&AS 109, 447).

Results of the EUVE survey are published by two source catalogues (ApJS 93, 569; 102, 129), including positions, count rates, and optical counterparts for a number of cataclysmic and X-ray systems. Another EUV allsky survey was conducted with the ROSAT Wide Field Camera: data on about 80 binaries are found in the 2RE Source Catalogue (MN 274, 1165); optical identifications include several new magnetic cataclismic variables and white dwarf/M star pairs (MN 274, 1194). 
More specific information on the Fe feature seen in the 6-7 keV spectra of $31 \mathrm{X}$-ray binaries observed with EXOSAT is given in A\&AS 109, 9. An atlas of high resolution line profiles of 94 symbiotic systems are published in two part atlas (A\&AS 102, 401; A\&AS 103, 201). Chromospherically active binaries are catalogued in A\&AS 100,173, where data on orbital elements and physical properties for 206 spectroscopic binaries (SB) are summarized. Finally, orbital elements of 24 binary pulsars can be found in a catalogue of 558 pulsars (ApJS 88, 529).

Among specific subgroups of CBS, especially cataclysmic variables and chromospherically active binaries have attracted continued strong attention.

Cataclysmic variables (CV). Relevant studies and data collections include: colours of 179 CVs (A\&AS 104, 79) and 65 old novae (AJ 108, 639) covering long time spans; sampled CCD spectrophotometry in the $3300-9150 \AA$ range for 25 faint systems (A\&AS 114, 575); the Tololo Nova Survey concerning intrinsic reddening in postoutburst novae due to dense globules, ejecta masses, and spectra (ApJ 426, 279; ApJS 90, 297); element abundances in optically thin nova shells of a sample of novae with outbursts between 1978 and 1989, which are compatible with the thermonuclear runaway model (A\&A 291, 869); near-IR photometry of the secondaries of old novae and mass transfer rates (MN 266,761); a statistical study of 1200 IUE spectra of 36 dwarf novae in quiescence for testing the accretion disk model (A\&A 281, 759); accretion disk radii of 35 eclipsing CVs showing systematic differences between CV subclasses (MN 279, 219).

Chromospherically active binaries (ChAB). Many statistical studies and surveys focussed on binaries exhibiting chromospheric, coronal and star spot activity, a few of which are briefly mentioned below.

The space density of active binaries as inferred from an EINSTEIN Xray survey is found to be lower than previously assumed, while the median $\mathrm{X}$-ray luminosity is higher (A\&A 298, 482). Simultaneous VLA, IUE, and ROSAT observations were used to search for correlations between radio vs soft X-ray coronal and chromospheric emission (A\&A 284, 91). From a large sample of RS CVn systems detected with ROSAT PSPC an X-ray flux - rotational period relation was found for evolved stars (ApJS 86, 599). CORAVEL high precision rotational velocities were measured for many active binaries with evolved components and were discussed concerning the link between rotation and coronal activity (A\&A 302, 745).The question of coplanarity of rotational and orbital planes in asynchronously rotating and tidally locked long-period RS CVn systems has been readdressed (ActaA 44, 33; ActaA 44, 393): contrary to asynchronous cases, the synchronous systems have rotational axes perpendicular to the orbital planes. High resolution $\mathrm{Ca}$ II $\mathrm{H}$ and $\mathrm{K}$ and $\mathrm{H} \alpha$ line profiles have been measured and analyzed 
to study the physics and geometry of chromospheric activity regions (A\&AS 103, 413; A\&A 274, 373; ApJS 96 81; A\&A 283, 179) .

Additional statistical studies and surveys of special CBS subgroups are quoted in the appropriate chapters of this report. Useful bibliography on CBS and related topics can be found in the Bibliography 8 Program Notes on Close Binaries (BPN on CBS), which can now be retrieved electronically from http://www.sternwarte.uni-erlangen.de .

\section{OBSERVATIONAL DATA}

\subsection{Newly Discovered Systems (Giuseppe Cutispoto)}

A large number of papers containing new observations of CBS was published in the last triennium. It is obviously impossible to report in detail on each newly discovered systems. Updated references and data can be retrived via WWW at the centres quoted in the previous chapter. Moreover, the Astronomy $\mathcal{O}$ Astrophysics Abstract (Springer-Verlag) and the Astronomy and Astrophysics Abstract Service at Harvard Obs (http://adsabs.harvard.edu/abs. $h t l m$ ) offer a precious service to astronomers. Hence, the present report will only try to illustrate general trends on CBS studies.

One of the highlights has been the increasing interest in the optical identification and study of low mass and CV X-ray binaries discovered by the EINSTEIN, EXOSAT, ROSAT, GINGA and EUVE observatories. The pubblication of large lists of X-ray/EUV sources (MN 260, 77; AJ 107, 751; MN 274, 1165; A\&A 297, 764; A\&AS 115, 41) has triggered optical studies that have, in turn, led to the discover of a number of active CBS (A\&A 281, 756; A\&A 297, 770; MN 274, 1194; AJ 111, 439; MN 276, 397; IBVS 4140; ASP Conf.S 64, 98; PASP 106, 822; ApJ 461, 951; ASP Conf.S 64, 551; A\&AS 115, 41) and CVs (MN 272, 531; MN 273, 711; MN 264, 132; ApJ 440, 834; PASP 106, 209; A\&A 297, L37; BAAS 26, 1346).

Photometric observations have been increasingly performed by unmanned Automated Photoelectric Telescopes (APTs), which have proved to be very effective in the discovery of new systems (AJ 110, 2926; A\&A 281, 756; IBVS 4114), and particularly suitable for long-term studies (JApA 15, 321; ASP Conf Ser 64, 483; 105th ASP Meet, p. 277).

Multisite and multiwavelength studies have been successfully performed and have clearly demonstrated their important role also in promoting specific research projects. Typical programs include photometry and spectroscopy from ground-based and space-born telescopes, the monitoring of selected targets at different wavelengths over a large number of consecutive orbital/rotation periods. This allows us to identify and disentangle permanent from transient features and to probe the vertical structure of stellar 
atmosphere (AJ 443, 319; PASP 107, 232; A\&A 298, 505; MN 271, 981; ApJ 435, L141; AIP Conf Proc 280, 324; ApJ 424, 967; IAU Symp 162, 206).

The new CBS listed below certainly deserve additional and extensive observations: 7 Cam (IBVS 4176); LL Vel (A\&A 294, 190); ADS 11060 (AJ 108, 694); BD+08 102 (ApJ 438, 364; IAU Symp 176, Poster, p.99); HD 9902 (A\&AS 115, 41); HD 30957 (AJ 108, 1936); HD 80492 (BA\&AS 185, 851); HD 21155 (IBVS 4008); HD 43478 (IBVS 3978); HD 100022 (A\&A 306, 278); HD 181131 (A\&A 299, L29); HD 183133 (MN 266, 740); HD 220820 (Obs 116, 17); SAO 130113 (A\&AS 115, 41); SAO 202618 (A\&AS 115,41 ); 1E0315.7-1955, 1E0505.0-0527 (A\&A 306, 278); 1H 1752+081 (ApJ 424, 955; A\&A 288, 204; MN 273, 17); 2S 0114+65 (AJ 109, 396); EC 11575-1845 (MN 275, 100); EUVE 0720-317 (ApJ 433, L29); GL 410 (IBVS 4091); G102-21 (A\&A 298, 505); H 0459+246 (ApJ 428, 785); H 0857-242 (ApJ 428, 785); KUV 0859+415 (AJ 108, 214); RE 0720-318 (MN 273, 76); RE 0751+14 (MN 266, 703; 271, L25; 271, 372); RE 1307+535 (MN 270,$650 ; 271$, L41); RE 1738+665 (MN 271, 175); RX J0019.8+2156 (A\&A 294, L1; A\&A 294, L5); RX J0422+32 (MN 274, 565); RX J051541+0104.6 (ApJ 440, 834); RX J0558.0+5353 (A\&A 291, 171); RX J0929.1-2404 (MN 281, 808); RX J19402-1025 (MN 273, 681; PASP 107, 307).

\subsection{New Ephemeris and Period Variations (Carla Maceroni)}

The catalogs of eclipsing binaries obtained as by-products of the microlensing searches contain by far the largest collection of new ephemeris published in the last three years.Limiting ourselves to public domain catalogs, the OGLE survey yielded 1281 binaries with $0^{d} .7<P<115^{d}$ from twelve 15 'x15' fields in the Baade's window (see ActaA 45, 433). The EROS collaboration yelded 78 new eclipsing binaries with $0^{d} .7<P<50^{d} \mathrm{~d}$ in the LMC (A\&AS 109, 447). Though these determinations have a lower than usual precision, they have the great advantage of homogeneity and number.

The need of processing great number of variable light curves required the development of new methods of period determination (A\&A 289, 404; ActaA 44, 317). Similar methods yielded the periods of a number of CBS discovered in open and globular cluster (see ASP Conf Ser 90,270) which are promising tools for period evolution studies.

A few tens of new or improved periods mainly of field stars have been published in the IBVS alone. Many others appear in more complete photometric studies of CBS. Even more numerous are the measurements of eclipse times from the same sources. Those papers cannot be individually referred here. A list of eclipse times and updated ephemeris is regularly published in Rocznik Astronomiczny (Cracow Obs). 
The period variations of 6 late-type CBS and the related physical mechanisms affecting the system geómetry have been analysed (A\&A 293, 371). Systematic studies of light and period variations have provided evidence of solar-type magnetic cycles in the late components of a variety of close systems. A correlation between the rate of RS CVn period change over 35 years and the migration rate of its photometric wave has been interpreted in terms of the Applegate's model (see $\S$ 6.2). A similar explanation was suggested for V471 Tau (A\&A 281, 811). From the long term luminosity variation of four well observed CVs in the AAVSO database it appears that the amplitude variations and timescales are consistent with solar type cycle models (PASP 106, 1075). The same model has been applied to the orbital period change of the eclipsing pulsar binary PSR B1957 +20 (ApJ 426, L85; ApJ 436, 312). Evidence of magnetic activity in Algols, including period changes, has also been found (ApJS 88, 199).

The question of period changes related to apsidal motion in eccentric CBS is addressed in $\S 5$.

\subsection{Optical Photometry (Francis C. Fekel)}

As evidenced by the number of papers listed in the BPN on CBS the discovery and analysis of $\mathrm{CV}$ and pre-CV binaries continues to be a major area of interest. The proceedings of the conference on Cataclysmic Variables (Abano Terme, Italy) contain several papers discussing photometric observations. Photometry of CVs is also extensively interwoven into the recent book Cataclysmic Variable Stars (Cambridge Univ Press). Newly identified systems include RX J1940.2-1025 (MN 273, 681), RE 0720-318 (MN 273, 711), and EC 11575-1845 (MN 275, 100).

Candidates for a system consisting of a Wolf-Rayet star plus compact object were observed at high time resolution to search for pulsed radiation from possible neutron star companions (AJ 108, 678). Of the 10 candidates observed, a claimed period for HD 96548 was not confirmed, and only EZ CMa show evidence for a possible period of $0.11 \mathrm{~s}$. Thus, with the exception of Cyg X-3, observational evidence for this evolutionary stage of massive CBS is still lacking.

CCD photometry continues to be used as the major method of discovering many short-period eclipsing binaries in clusters: M71 (AJ 108, 1810), NGC 3293 (MN 267, 1060), NGC 4372 (MN 264, 785), NGC 4755 (MN 267, 1071), NGC 6791 (MN 265, 34), NGC 7789 (A\&A 295, 101), and Collinder 261 (MN 273, 59).

The NATO-ASI workshop on The Impact of Long Term Monitoring on Variable Star Research (Ghent, Belgium) included several papers on photometry of Barium, S-stars, symbiotics, and contact binaries. Some of 
this data are available in the latest catalogs of long-term photometry of ESO (A\&AS 109, 329; A\&AS,113, 31).

Optical follow-up observations of sources discovered by the EXOSAT, ROSAT, and EUVE satellites have shown that the lists of such sources are dominated by late-type active stars.

Understanding the phenomena produced by enhanced chromospheric activity in stars, many of which are in binary systems, remains an important field of study. Because of their complementary nature (ApJS 222, 181), the use of optical photometry in conjunction with high-dispersion spectroscopy is becoming increasingly prevalent in the analysis of surface features: e.g., HD 106225 (A\&A 281, 395) and RS CVn (AJ 106, 1181). Such a combination is particularly needed for surface imaging of late-type contact binaries. Analysis of AE Phe and YY Eri (A\&A 288, 529) showed that Doppler imaging can be applied once photometric solutions give information about the shapes and surface brightness distributions of the stars.

A number of photometric results were presented at IAU Symp 176 on Stellar Surface Structure (Vienna, Austria). Long-term optical monitoring of a variety of active binaries has continued (A\&AS 102, 655; A\&AS 111, 507). More extensive long-term studies have been made of RS CVn (A\&A 301, 75; AJ 109, 2169), $\lambda$ And, $\sigma$ Gem, II Peg, V711 Tau (ApJS 97, 513), SS Boo, MM Her (AJ 109, 2169), and UX Ari (A\&A 301, 788). A long term study of XY UMa (MN 277, 747) provided evidence of a polar spot on the hotter star. Fifteen years of photometry have been analyzed for HR 7275 (A\&A 282, 535). Multisite photometric campaigns have been carried out on V711 Tau (A\&A 282, 168) and ER Vul (A\&A 291, 110). A three-spot solution was required to fit the 1991 light curve of II Peg (A\&A 299, 115), and for the first time spots with significantly different mean temperatures from one another were found. While most photometric analyses find that 2-spot fits characterize the observations, independent analyses with different methods of RS CVn (AJ 106, 1181; A\&A 301, 75) indicates several moderate-size spots. A different spot scenario, the coalescence of random spots (ApJ 462, 888) was examined by comparing calculated long-term light curves with long-term photometric observations of several chromospherically active binaries. Properties of the calculated curves seemed to mimic some of the real data sets quite well. Metal-poor binaries are also being surveyed to investigate the properties of the dynamo in a metal-poor environment (AJ 110, 2926).

A search for optical flares on 64 chromospherically active binaries (PSAP 108,242 ) resulted in the detection of flares on only UX Ari, II Peg, and AR Psc. II Peg is a favorite target for flare monitoring (MN 270, 427; A\&A 299, $115)$, but its rate appears to be variable. Properties of the monster optical flare on V711 Tau were analyzed (ActaA Sin 34, 245). 


\subsection{Ground-based Spectroscopy (Ronald W. Hilditch)}

A survey of papers, published in the interval 1993 July - 1996 June, which contain new ground-based spectroscopic data, shows a significant reduction in the numbers of papers over the previous triennium, perhaps counteracted by an increase in the sophistication of both the observing techniques and the data analyses employed.

The subgroup of binary stars that has been studied most intensively is the cataclysmic variables, by which I mean all short-period, post-masstransfer, evolved systems with at least one degenerate component. Allocations of $4-\mathrm{m}$ telescope time in sets of 3-4 nights have been exploited fully with spectroscopic data being obtained at moderate resolution $(2 \AA)$ and $\mathrm{S} / \mathrm{N}$, but with clever control of the CCD integration and read-out sequences to secure spectra at time resolutions of only a few seconds. Combined with high-precision optical or X-ray photometric ephemerides, such sequences of spectra have been used in studies of intermediate polars to separate phenomena associated with the spin period of the degenerate star (tens of seconds) from those associated with the orbital periods (tens to a few hundred minutes) (cf. MN 269, 517; A\&A 282, 493). Accretion discs in nova-like CVs and streams in polars have received equally intensive treatment, with Doppler-imaging techniques being utilised to establish spatially-resolved spectra across the discs (A\&A 283, 441), and the locations of the main sources of emission from accretion streams or curtains (A\&A 283, 508).

Doppler-imaging and tomographic separation of spectra have also dominated the studies of classical Algol systems (ApJ 414, 830; ApJS 88, 199; ApJ 438, L103), showing details of the accretion stream morphology, and further evidence of magnetic activity on the late-type secondaries. Careful use of cross-correlation procedures to establish more reliable radial velocities of both components in Algol systems has also improved the tests of observational data against evolutionary models (cf. A\&A 311, 567). Whilst such procedures have also been applied to the active WUMa-type contact binaries (e.g. A\&A 288, 529), it is disappointing to record a marked decrease in spectroscopic studies.

Results on massive binary systems containing $O$ stars have progressed substantially thanks to the applications of tomographic separation of spectra (ApJ 423,446) and disentangling algorithms (A\&A 281, 286). These procedures require spectra that are evenly distributed across orbital phases in order to be most effective, but the resultant radial velocities do seem to be free of the long-standing difficulties of spectral-line blending in such systems. The number of O-star eclipsing binaries with masses determined to a precision of 2 per cent is beginning to increase beyond two! 
Of all the spectral lines available, the most popular in the last 3 years have undoubtedly been $\mathrm{H} \alpha, \mathrm{Li} 6707 \AA$ and the Raman-scattered lines at $6825 \AA$ and $7082 \AA$. There are many studies of the chromospherically active RS CVn and BY Dra binaries, involving simultaneous optical and UV spectroscopy and photometry, investigating the rotational modulation of starspot diagnostics and the links between these and the strength of the $\mathrm{Li}$ line. The polarised behaviour of the $6825 \AA$ and $7082 \AA$ lines in symbiotic systems is being exploited to determine the orbital characteristics of these long-period systems (e.g., A\&A 310, 235) independently of the usual radialvelocity studies. Li line studies appear also, of course, in investigations of pre-main-sequence (PMS) binaries, which in turn are being discovered and studied more intensively now (cf. Eighth Cambridge Workshop, p. 578) and used as tests of star formation.

In addition to all these detailed investigations of strongly interacting binaries, there is almost as much activity (in terms of published papers) on longer-period systems ( $P>100$ days, say). Almost invariably, these studies are being made by investigators who have their own facilities that can accommodate long-term observing programmes spanning years. We see radial-velocity curves that are beautifully defined, with orbital phases reobserved on different nights to ensure genuinely independent observations. The range of velocity variations is, of course, much smaller in these longerperiod systems, and great care must be exercised in securing velocities that are unaffected by systematic blending effects. A major step forward in this endeavour has been the development of TODCOR (ApJ 420, 806 and subsequent papers) providing the methodology to use two spectral templates simultaneously in the determination of radial velocities of binary stars with components of differing spectral type. These developments are extending the capability of radial velocity work in double-lined systems to smaller amplitudes, and closer to the systems that have sufficient separations for speckle interferometry. But the results that gave this reviewer most pleasure came from the application of TODCOR to the short-period $(\mathrm{P}=1.27$ day) eclipsing binary containing two dwarf $M$ stars, CM Dra. The masses of the two stars in this system are now determined to a precision of 0.001 solar masses, which allows them to be used as tests of the primordial helium abundance.

\subsection{UV Flux and Spectroscopy (James E. Neff)}

Ultraviolet observations are crucial for studying several phenomena that are either unique to or enhanced in close-binary systems. These include magnetic activity in the outer atmospheres, interacting magnetic fields of the two components, and mass transfer between the components. Ultravio- 
let observations are also used to study the fundamental properties of white dwarfs in CBS.

For years the workhorse of ultraviolet spectroscopy has been the International Ultraviolet Explorer (IUE). From its vantage point in geosynchronous orbit, IUE has been used to study individual systems continuously for several days. This has enabled studies of variable phenomena in CBS over their rotational and orbital time scales. The IUE era is now winding down, but the extensive archival data base is being reprocessed to enhance their quality (AJ 111, 517). This data base will enable studies of variabilitty over much longer timescales. The Hubble Space Telescope (HST) will permit more detailed studies at higher spectral resolutions and higher S/N. Some preliminary results were reported in Science with the Hubble Space Telescope II (ESA, ST-ECF), and the Second GHRS Science Symposium (ASP Conf Ser) contains several review papers that cover ultraviolet observations of CBS with HST.

Ultraviolet emission from the outher atmospheres of active stars traces the morphology of the magnetic field in these systems, which is enhanced by the rapid rotation induced through spin-orbit coupling. By studying rapidly-rotating stars in CBS with IUE over rotational timescales, we have been able to map the magnetic structure in their outer atmospheres. Similar programs can be conducted with HST, but its low-earth orbit renders it much less efficient. Taking advantage of a continuous viewing zone to observe V824 Ara throughout a full orbit in May 1996, maps of the transition region (from C IV lines) and, for the first time, some information about the coronal structure (from Fe XXI lines), as well as a chromospheric map (from $\mathrm{Mg}$ II lines) were derived. Ultraviolet surface imaging results were reviewed at the conference on Stellar Surface Structure (Vienna, Austria). The analysis of emission line profiles obtained with HST require more complex models than those developed for IUE, because the line profiles often show extremely broad wings (ApJ 458, 761).

The next three years will see more detailed studies of ultraviolet line profiles conducted with HST using the Space Telescope Imaging Spectrometer. Chromospheric imaging should approach the sophistication of photospheric (Doppler) imaging. Tomographic studies of cataclysmic variables will be possible using a host of lines heretofore unavailable, and high timeresolution studies using atmospheric eclipses will provide unprecedented information on the magnetic structure of stellar atmospheres and on the circumstellar environment in CBS stars. 


\subsection{EUV Flux and Spectroscopy (Andrea K. Dupree)}

This report marks the beginning of a rich new field of extreme ultraviolet spectroscopy of CBS, and initial publications show that revisions of previous concepts are necessary, and outstanding problems remain. Three conference proceedings include reports of much current work: Cool Stars, Stellar Systems, and the Sun: Eighth Cambridge Workshop (CS8) (ASP Conf Ser 26), Astrophysics in the Extreme Ultraviolet (IAU Coll 152), and the Ninth Cambridge Workshop (CS9) (APS Conf Ser).

EUV surveys carried out by ROSAT and EUVE showed that CBS containing cool star components are strong sources of extreme ultraviolet emission (MN 260, 77; MN 274, 1165; ApJS 93, 569; ApJS 102, 129). A comparison of the two EUV catalogues can be found in MN 273, 93. In the Second EUVE source catalogue $37 \%$ of the sources are F, G, K, and M stars (IAU Coll 152,5$)$ and of these $\approx 80$ systems are of RS CVn-, W UMa-, and BY Dra-type. Such systems are strong EUV sources since they have high temperature coronae and are relatively close by, so that many of them have been measured spectroscopically in the EUV region.

From the very first results it was apparent that the spectrometers on NASA's EUVE can obtain superb spectra of cool stars. Rich emission lines, predominantly from iron, dominate the spectra and yield unique and explicit information on the temperature structure of their coronas. The wellstudied spectra of systems such as Capella, $\lambda$ And, UX Ari, AR Lac, HR 1099 (=V 711 Tau), VY Ari, 44i Boo (ApJ 418, L41; A\&A 302, 438; IAU Coll 152, pp.89, 129, 105, 81) show several interesting characteristics: a continuous distribution of plasma temperatures is present between $10^{4}$ and $10^{7.2} \mathrm{~K}$, thus previous simple 1-temperature or 2-temperature models do not adequately represent the corona for such systems. The emission measure distribution (EMD) has a clear minimum near $10^{6} \mathrm{~K}$ which differs from the solar example, and contradicts our understanding of the location of the maximum radiative losses of a collisionally dominated plasma in equilibrium. A narrow enhancement in the EMD is present at $10^{6.8} \mathrm{~K}$ (the bump); this feature is relatively constant with orbital phase. Electron densities are high $\left(10^{12}-10^{13} \mathrm{~cm}^{-3}\right)$ at temperatures of $10^{7} \mathrm{~K}$, as inferred from $\mathrm{Fe}$ XXI line ratios in many of the systems. Rotation appears to be the significant physical parameter in producing an enhanced emission measure feature occurring over a narrow temperature range. This feature is generally present in both rapidly rotating single stars and those systems with periods $\lesssim 13$ days of the binary systems. When the emission measure is evaluated with the high measured density from EUVE line ratios, a small scale for the emission results - typically much less than a stellar radius.

The values of coronal abundances inferred from EUVE spectra remain 
puzzling; AR Lac (IAU Coll 152, 129), CF Tuc (ApJ 464, 898), Algol (ApJ $444, \mathrm{~L} 45$ ) indicate a reduced iron abundance, yet Capella (which has the strongest and highest quality spectra) shows normal solar photospheric values of iron (IAU Coll 152, 105). To date, the spectrum of only one single star ( $\epsilon$ Eri), appears consistent with the solar pattern of enhancement of the first ionization potential ions (the FIP effect) (ApJ 462, 948), and Procyon appears to have solar photospheric abundances (Science 267, 1470; ApJ 443, 393).

Variability of the EUVE emission flux, both on an orbital time scale and the more rapid typical flaring events, have been found in many systems, i.e. CF Tuc, HR 1099, Capella, UX Ari, AR Lac (IAU Symp 176, 89; ApJ 421, L43; ApJ 464, 898; IAU Symp 176 Posters, 184; MN 275, 1232). Finally, the orbital behavior of EUV emission in AR Lac suggests the presence of extended structures (IAU Coll 152, 129).

\subsection{Polarization Studies (Robert H. Koch)}

Non-visible-band polarization is becoming more common. Results from the spacecraft UV are forecast by papers in PASP 106,1019 and BAAS 28, 912, and a more extended evaluation of the interstellar polarization spectrum is summarized in BAAS 28, 914. Already several CBS have been observed. New instruments also operating in the X-ray region and at $1200 \mathrm{~nm}$ have both been described in BAAS 28, 912. From several examples below, it may be inferred that spectropolarimetry is becoming more and more the tool of choice and has even been applied to faint objects such as $\mathrm{N}$ Cas 1995(BAAS 28, 913). Three miscellaneous methodological advances may be noted: a statistical critique of some parameters of the Glasgow Model (MN 267,5) and the question of dealing with detached, finite-radius stars in a scattering envelope (ApJ 432, 262). Finally, attention has been called to possible polarization pulses at caustic crossings when a background star is lensed by a CBS (MN 279, 571). The rest of this review is ordered largely by stages of binary star evolution. If FU Ori-type variables really represent episodes in star formation, the results concerning Z CMa (ApJ 417, 687) are worth noting: an unseen $\mathrm{Ae} / \mathrm{Be}$ companion is suggested from polarization evidence. Much more graphically, the VLA/VLBI linear and circular measures (AJ 111, 918) of the composite T Tau-type pair HD 283447 lead to a picture of tangled magnetospheres from the two K-type stars, each of which displays solar-type radio variability. Apparently only two papers are concerned with polarization from MS binaries: a restudy of old measures of Y Cyg (PASP 106, 370) concluding that long-term sporadic variability dominates the behavior of the system; and the indirect evidence of intrinsic polarization for CW Cep (Obs 106, 89). 
Extended monitoring of 20 spotted-star binaries was accumulated by several workers (AZh 70,1322; A\&AS 102, 343). A number of these objects are clearly polarization variables. The reversal with frequency of the circular polarization state of RS CVn-type systems suggests 2-component emission in the systems' coronae (ApJ 444, 342). For conventional mass-losing binaries a major development appeared in the coupling of a polarizationanalysis piece of software to both the Glasgow model and to the familiar Wilson-Devinney code hitherto used only for light- and velocity-curve analyses. So far, this has been applied only to $\beta$ Per (ApJ 413,670) with only a partial, but very promising success. Of other systems, only for HD 207739 (AZh evaluated. Efforts for 32 Cyg (MN 278, 975) and RY Sct (ApJ 439, 417) contain mostly indicative results. There are also generalized models for symbiotic variables which are applied to variable polarization across Raman-scatterd lines in the spectra of V1016 Cyg and SY Mus (A\&A 281, 145; A\&A 310, 211; A\&A 310, 325) and the WR-component binaries EZ $\mathrm{CMa}$, HD 97152, HD 50896, and HD 152270 (ApJ 413, 724). By concurrent polarization measures in the continuum and within selected emission lines it is shown that the electron-scattering shells are essentially spherical. Analysis of GP CepA (AZh 70, 319) and V 444 Cyg (OAP 7, 313) have become available (see also IAU Symp 163).

Major results on polarization for neutron-star systems include: an upper limit to the intrinsic polarization of Cyg X-2 (A\&A 296, 390), evidence of polarization intrinsic to Cyg X-3 (AJ 108,605), a 1011 G field for EXO $2030+375$, albeit without any real modelling (MN 271, 981), and intrinsic variability for $4 \mathrm{U}$ 0900-40 (ApJ 457, 859). White dwarf systems are represented by the seemingly unpolarized condition of IP Peg (MN 274, 27), by the null observations for KUV $0859+415$ (AJ 108, 214), and by the prose description of WGA J1802.1+1804 (ApJ 455, L43). But the real testimony to interest in these systems is shown by the repeated invocations of Glasgow-type analyses or more detailed models (MN 251, 28).

A number of binaries have been analyzed to evaluate the orbital and magnetic and spin-pole inclinations, the electron temperature, opacity, and the 2-D distribution of the magnetic field and its mean field strength: $B Y$ Cam (A\&A 283, 163), V1500 Cyg (ApJ 441, 1995), Cyg XR-1 (ApJ 457, 859), X Per (PASJ 47, 89), 1H 1752+081 (MN 273, 17), RE 0751+14 (ApJ 410, L107), RE 1307+535 (MN 271, L41), RE 2107-05 (ApJ 424, 967), RE J1844-741 (MN 273, 579), RE J1938-461 (MN 268, 128), RX J0515.6+ 0105 (MN 275, L61), RX J1712.6-2414 (MN 275, 1028), 4U1700-37 (ApJ $457,859)$. 


\subsection{Highlights of Radio Binary Observations (Barry Geldzahler)}

Once again I have been given the bittersweet task of summarizing the highlights in radio binary studies for the past three years. It is an honor to do so, but because of space limitations, I have been forced to omit many fine studies by many fine researchers.

VLBI observations of Algol. VLBI data from the literature have been reanalyzed (ApJ 410,808) to study the dynamical evolution of Algol. A unique relative inclination of the orbital planes of the inner and outer binaries $\left(i_{\text {rel }}=90.3 \pm 0.3\right.$ degrees $)$ was found. Three point mass simulations of the Algol system parameters yield the surprising result that the inner binary eccentricity should increase to 0.9992 in just over 1000 years! Further simulations showed this result is typical broad patch of phase space, even for triple systems with large period ratios. This suggests that for the Algol system the non-point-like nature of the $\mathrm{K}$ star suppresses the catastrophic implications. However, for systems with smaller ratios of component size to separation (both triple stars and planets in binary star systems), this new effect may have a significant impact on orbital parameters, or even induce collisions.

The Wolf-Rayet binary WR140 $=$ HD193793. Eight years of VLA data of WR140 (WR+O), which has a 7.9y highly elliptical orbit, have been obtained (ApJ 451, 352). Its variable, non-thermal emission is accounted for as synchrotron radiation from relativistic electrons accelerated in the shock between the WR and O-star winds. This system is an ideal laboratory for studying the properties of hot stars and the physics of particle acceleration by strong shocks in the winds. The VLA observations densely cover every phase of the orbit and extend our knowledge of the system to $2 \mathrm{~cm}$, the shortest wavelength every applied to this system. The standard model of spherically symmetric stellar winds colliding cannot easily account for the observational data. Instead, a model wherein the WR wind is strongest in the equatorial plane and the mass is confined to a plane is consistent with the observations. The model has the added attraction of accounting naturally for sudden formations of dust.

VLA and VLBA observations of Sco X-1. Both intrinsic variability and astrometric studies of the 18 hour period, low mass X-ray binary Sco X-1 have been continued. Using VLA data from six epochs between 1981 and 1990, strong evidence was found for 3 hour periodic radio emission emanating from the system. The apparent periodicity is evident in each epoch when radio emission from Sco X-1 is about the detection threshold. The binary period is not known well enough to connect the phases between the epochs. Finally, three modes of radio variability were reported: slow, intermediate, and rapid time scale of hours, tens of minutes, and minutes. 
The rapid time scales set a light travel time size limit of 1 au on the size of the radio emitting region for a distance of $200 \mathrm{pc}$.

A 200 pc distance for Sco X-1 has been derived from published VLA observations (ApJ 383, 289). VLBA observations are expected to improve this $3 \sigma$ result by an order of magnitude. Sco X-1 is the only X-ray binary for which there is a reliable distance determination, a key parameter for models of low mass X-ray binaries.

NRL/Green Bank interferometry of Cyg X-3. Quenched quiescent flux densities prior to radio outbursts have been reported (AJ 108, 179; AJ 110, 290). The quenched radio emission persists on a longer timescale than is explained by the early self-absorbed stage in existing models. From a comparison of GINGA ASM X-ray and GBI radio data (AJ 108, 179; AJ $110,290)$ it was found that the radio flares occur in the X-ray high, soft state.

The NRL Group monitors the flux densities of a host of radio binaries including Algol, HR1099 (= V711 Tau), and LSI +61 303 = 0236+610.

\subsection{Radio Flares (Grazia Umana and Corrado Trigilio)}

Monitoring programs. A systematic radio monitoring of the brightest RS CVn CBS is carried out at CNR VLBI station in Noto (Italy) with a 32-m telescope. This program aims at studying the long-term behaviour and flaring episodes of active systems.

From the first three years of single-dish monitoring, HR 1099 (V711 Tau) appear to undergo extended periods of strong activity, alternate to periods of low flux density level (A\&A 298, 143). Moreover, using additional data from the literature, a correlation between the beginning of a new activity cycle, as suggested by long-term photometry, and the occurrence of strong radio flares is apparent.

At the end of 1992 UX Ari underwent a period of strong activity, extremely and unusually long, which was observed from Bonn (A\&A 278, L51; Radio Emission from the Stars and the Sun, ASP Conf Ser, p. 330), Noto (ditto, p. 342) and VLA (ApJ 439, 983). A dependence of strong flaring activity on the orbital phase was found. This suggests the existence of compact coronal active layers associated to starspots groups.

Coherent emission from RS CVn systems. Highly variable, on very short timescale, and highly polarized radio flares have been observed in several RS CVns (A\&A 286, 181; ApJ 444, 342). These flare events, which are confined to lower frequencies (below $2 \mathrm{GHz}$ ), are attributed to mechanisms such as cyclotron maser emission or plasma emission. The coherent emission is polarized in the sense opposite to the polarization measured at higher frequencies. Therefore, it is responsible for the polarization reversal 
observed in the radio spectra of active binaries, which is otherwise very difficult to explain with the standard gyrosynchrotron models.

Superluminal motion in our Galaxy. Immediately after a strong flaring event on GRS 1915+105, detected with VLA in March 1994 (Nat $371,46)$, a jet-like structure was discovered showing radio knots emerging in opposite directions from a compact variable core and moving at superluminal speed. GRS $1915+105$ is the first superluminal source where the proper motions of the approaching and receding radio knots can be detected and measured.

\subsection{Imaging Close Binaries with Interferometric Telescopes (Yoji Kondo and Harold A. McAlister)}

In this report radians (instead of arcseconds) will be used to express resolution; by multiplying the distance (in $\mathrm{km}$ ) to a binary by the fractional radian, the resolution (in $\mathrm{km}$ ) at the binary is obtained.

The Center for High Angular Resolution Astronomy (CHARA) at Georgia State University is building a 5-telescope optical/IR interferometric array with a limiting resolution at $500 \mathrm{~nm}$ of 0.2 milli-arcsecond (mas) approximately $10^{-9}$ radian - and 1.0 mas at 2.2 microns resulting from the longest baseline of approximately $350 \mathrm{~m}$. The telescopes comprising the CHARA array have apertures of $1.0 \mathrm{~m}$ and will be arranged in a Yshaped array to give two-dimensional, non-redundant $(u, v)$-plane coverage required for imaging. The facility will be constructed on Mt. Wilson where it can take advantage of the site's excellent seeing conditions. Under subarcsecond seeing, the Array should have limiting magnitudes of $\mathrm{V}=+10$ and $\mathrm{K}=+15$. The incorporation of wave-front correcting adaptive optics at a future date could push the V limit to +13 . The Array will have broad capabilities in stellar astrophysics, and will resolve the majority of the spectroscopic binaries in the northern hemisphere. The individual photospheres of many of these components will also be resolved. A 2 solar-mass binary at a distance of $100 \mathrm{pc}$ would be resolvable for orbital periods exceeding 12 hours. Construction on Mt. Wilson will take place during 1996-98 with first fringe anticipated for early 1998.

At NASA, interferometric technology for free-flying satellites will soon be under test. In order for this technology to work, we need to know the relative positions of the satellite telescopes within some tens of Angstroms for the visible light. When this technology is perfected, we can in principle have any length of base-line we want for interferometry, which will mean virtually unlimited resolution. We currently have a plan to test a pair of interferometric telescopes in orbit in the year 2000. In space, there is no need to correct for atmospheric distortions. 
Even before the availability of this new interferometric technology for orbiting telescopes, the Moon can provide an excellent site for interferometry as it provides a solid base for the telescopes. At a separation of 350 $\mathrm{km}$, we can improve the resolution of the Mount Wilson telescopes by a thousand times. A resolving power of $10^{-12}$ radian means that a tenth of the solar diameter can be resolved at a distance of 10,000 light years in visible light; we can even observe gas flows within and out of binary systems. We will be needing greater light collecting areas as we extend our reach to fainter stars; a multiple mirror system, like the CHARA array, can resolve that problem.

\subsection{Microlensing Phenomena (Anatolj Cherepashchuk)}

Microlensing as astronomical phenomena were discovered in 1993 by two groups: MACHO (Nat 365, 621) and EROS (Nat 365, 623) on the background of LMC and later by OGLE group (ApJ 426, L69) on the background of the galactic bulge. The major theoretical results since 1993 are: the suggested observation of binary stars via microlensing and the expected light curve characyteristics (ApJ 407, 440), the interpretation of the MA$\mathrm{CHO}$ event in terms of microlensing evidence of long periodic binary (A\&A $289, \mathrm{~L} 31$ ), and the detailed analysis of the resulting light curve and color changes during microlensing in the case the background star is a binary (AZh 20, L613; AZh 21, L263). Typical microlensing light curves for bulge background binary stars have been also calculated (A\&SSci 229, 265). In these papers the problem of detecting small celestial bodies like planets was discussed (see also ApJ 436, 112; BAAS 27, 1352).

The main theoretical results connected with polarization are the following. Polarization should be detectable during caustic crossing of a binary lens (MN 279, 571). This provides a new powerful tool of interpretation of binary lens. Others interesting results are connected with microlensing of one binary companion by the other: the effect is negligible in the case of ordinary stars and the probability of this event is $0.5-2 \%$ in the case of binary millisecond pulsars (ApJ 446,541). In the case of a wide binary lens, in which disconnected amplification regions emerge, there is a significant probability of repetition of amplification (ApJ 457, 93).

The main experimental results is the observation of light curves revealing the presence of binary lenses (ApJ 426, L69; A\&A 300, L17). The possibility of detecting binary star during microlensing observations has been also discussed (BAAS 26, 912).

An important by product of the microlensing observations program is the catalog of light curves of many thousands variables, including eclipsing binaries (BAAS 26, 912). 


\section{METHODS OF LIGHT CURVE ANALYSIS (Eugene F. Milone)}

Developments in light curve modeling have proceeded apace during the last triennium.

Linnell's code continues to be improved through the application of spectrum synthesis techniques to the analysis of binary systems (ApJ 434, 738; ApJ 459, 721), with application of the technique to EE Peg. The method, based on the Roche model, involves the use of a program package, which generates both a synthetic light curve and a synthetic spectrum, the latter for an arbitrary orbital longitude, that is physically self-consistent. The spectrum synthesis part of the package produces a system synthetic spectrum and separate component synthetic spectra which are the integrals of synthetic spectra appropriate to individual grid points on each component. The grid point spectra are characterized by the local values of effective temperature and log gravity. A $\chi^{2}$ test is used to fit the synthetic spectra to the observed ones, varying the polar effective temperatures as necessary to achieve a best fit. In the case of EE Peg, the process was used to derive the chemical composition of the primary. The synthetic spectrum program produces light intensities for 10 values of zenith angle at each wavelength. Consequently the spectrum fitting process produces exact values of limb darkening coefficients to use in the light curve solution(s). More recently, irradiated model atmospheres has been incorporated into Linnell's code, obviating the need for bolometric albedos. The part of the program package that treats accretion disks calculates monochromatic light curves as well as synthetic spectra from the two binary components, and from the face and rim of the accretion disk. The recent extension to incorporate irradiated model atmospheres applies to the case of accretion disk systems also. A further program innovation includes modelling of a hot spot on the accretion disk rim.

Various offshoots of the Wilson-Devinney program, the program of choice for this as well as the previous two triennia, have continued to be improved. One of these (wd93k93c) has developed diagnostic capabilities (see Close Binary Stars, p. 139; Pacific Rim Coll on CBS, p. 172). The application of the full range of Kurucz atmospheres and the computation of flux files for use with them has now been completed.

A new code based on the Roche model modified to take into account radiation pressure forces in addition to gravitational and centrifugal forces has been produced (A\&A 294, 723). The main problem to be faced in developing this code was that in this case there is no analytical representation of the potential field, and stellar surfaces must be computed by numerical methods. The same applies to the treatment of gravity brightening, for which the potential derivatives are needed. The resulting light curve so- 
lution code is based on the general logistics of the WD method, but uses the modified Roche model as underlying binary model, and the simplex method for parameter adjustment. The new code has been applied to the triple system IU Aur (A\&A 284, 853) and the O8 system AB Cru (A\&A 291, 185).

Recent improvements of Wilson's code are as follow.

1. Efficient computation of the attenuation of light by circumstellar gas for use in light curve fitting. The method is based on partly analytic treatment of attenuating paths through CS spheres. Arbitrary gas distributions are simulated by distributions of spheres.

2. Simultaneous pulse arrival time, light curve, and radial velocity analysis of X-ray binaries (The Evolution of X-ray Binaries, Am Inst Phys, $\mathrm{p}$. 483). One system that has been treated is Vela $\mathrm{X} 1=\mathrm{HD} 77581=\mathrm{GP}$ Vel.

3. Extension of light curve modeling to polarization modeling, including parameter adjustment (ApJ 413,670) for theory and application to Algol. Both circumstellar and photospheric polarization is included.

4. Development of another version of a light curve program to provide a major increase in precision, and improved convergence. Two reviews summarize these and other works (PASP 106, 921; IAPPP Comm 55, 1). Tables of limb-darkening based on the 1993 Kurucz atmospheres and for use with the enhanced WD codes have been computed (AJ 106, 2096). Limb-darkening, for use in EBOP and similar codes, has also been treated (A\&A 110, 329).

Finally, a new book on light curve modeling (Kallrath \& Milone, Modeling and Analysis of Eclipsing Binary Observations, Springer-Verlag, A\&Ap Ser) reviews past and present light curve solving methods and discussed options for the future.

\section{STELLAR PHYSICAL PARAMETERS (Alvaro Gimenez)}

During the last trienium important efforts have been dedicated to obtaining new accurate stellar dimensions from eclipsing binaries with double lined spectra. On the other hand, the generation of new stellar models (see A\&AS 109,$441 ; 114,549$ ) or global studies received slightly less attention than in the previous period. Interesting results and observational data were presented at the Calgary (Canada) conference on The Origins, Evolution, and Destinies of Binary Stars in Clusters (ASPC 90), and some discussions on physical parameters are included in the book The Realm of Interacting Binary Stars (Kluwer). New techniques were discussed in the Pacific Rim Colloquium (Seoul, Korea) on New Frontiers in Binary Star Research (pp. $1,127,154$, and 275). A new technique to disentangle composite spectra (A\&A 281, 286) has been applied to important hot binaries like V453 Cyg, 
DH Cep (A\&A 282, 93), and Y Cyg (A\&A 292, 507). Further, the use of ultraviolet radial velocities has proven very useful for the study of hot binaries like TU Mus (Obs 115, 317) or LY Aur (Obs 114, 107).

Of particular interest for stellar physical parameters, is the very high precision orbit of Capella obtained by long baseline interferometry (AJ 107, 1859) and the derived absolute dimensions of the component stars. Moreover, Spica was studied spectroscopically to determine new stellar dimensions (Astr Rep 39, 186) and compare with interferometric data.

Concerning systematic studies of CBS with the purpose of determining accurate stellar physical parameters, cross-correlation analysis has been adopted for the system V1425 Cyg (A\&A 276, 57) and CC Cas (A\&A 282, $455)$, and for a new Reticon radial velocity curve of the O-type system $Y$ Cyg (A\&A 297, 127). The group of the Copenhagen University has added the studies of the unevolved systems GG Lup (A\&A 277, 439) and AR Aur (A\&A 282, 787), the Ap system AO Vel (A\&ASS 109, 425) with eccentric orbit and a third component, V539 Ara (A\&A 308, 151) which contains a slow pulsating $B$ star, the evolved early A-type binary $\beta A$ ur (A\&A 291, $777)$ and the nearly contact system LZ Cen (A\&A 301,693). Published data on some faint secondaries in the spectra of the early B-type systems VV Ori, V380 Cyg, and V1765 Cyg (PASP 105, 721) and related questions of interest have been rediscussed (Obs 114, 1122).

Other detached binaries studied include AG Per (A\&A 291, 795), CO Lac (BA\&AS 27, 878), PV Cas (AJ 109, 2680), VV Ori (JApA 16, 407), V380 Cyg (AZh 73, 55), EM Car (Obs 114, 284) or EE Peg (ApJ 459, 721). In addition, the winds of hot CBS have been studied in the detached systems CW Cep (Obs 116, 89) and Y Cyg (Obs 114, 297).

Absolute dimensions have also been derived for the late-type binaries RT And and CG Cyg (AJ 108, 1091), cross-correlation radial velocities have been obtained for several RS CVn binaries (A\&A 305, 146) while a comparison with models has been made to derive stellar ages (A\&A 290, $137)$. The very low mass system CM Dra (ApJ 456, 356) has been analyzed and compared, together with YY Gem, against models (ApJ 451, L29). Moreover, the interesting system EK Cep, with a PMS secondary component, has been revisited from the point of view of new observations (Kin Phys Celest Bodies 9, 46), surface Li abundance (A\&A 274, 274), apsidal motion and the comparison with theoretical models (A\&A 302, 741).

Studies of orbit circularization and synchronization times has also progressed from the point of view of comparison with observational data for MS stars (A\&A 299, 724) and CBS containing giant stars (A\&A 296, 709). The asynchronous evolved system TZ For has been compared with theoretical models to understand its tidal evolution (A\&A 296, 180). On the theoretical side, studies on the orbital circularization in late-type binaries 
(ApJ 444, 338), the role of chaos in the circularization of tidal capture binaries (ApJ 450,722; ApJ 450,732), and the orbital evolution of highly eccentric binaries (ApJ 429, 632) have been published. Rotational velocities have also been analyzed (AcApSin 15, 57; 14, 228).

Some systems in open clusters have been reanalyzed using new observations, like V478 Cyg in Dol 42 (Kin Phys Celest Bodies 9, 39; Pis AZh 22,149 ), H235 in NGC 752 (AJ 109, 359) or EP Cep in NGC 188 (Ap\&SS $220,107)$. A full discussion of the possibilities offered by the study of binaries in clusters, the analysis of several individual candidates, as well as their contribution to understand stellar physical parameters is given in the mentioned ASPC 90 (pp. 109, 120, 135, 150, 152, 154).

The study of EBs in the Magellanic Clouds with the purpose of determining their absolute dimensions has continued on HV 1761 (MN 265, 189) with CCD contributions and new radial velocities for HV 5936 (MN 265, 1047) and some others (ApJ 437, 332). Reviews on the available information and possibilities for the near future are presented in ASPC 90 (p. 207 and 196) and ExA 5, 181.

The possibility of detached configurations for the classical semi-detached (s-d) systems RZ Oph (AJ 106, 754), WW And (AJ 106, 759) and S Cnc (AJ 106, 1162) have been discussed. The prototype system Algol has been analysed by cross-correlation techniques (A\&AS 101, 579). This bright star has also been used for high angular resolution interferometric measurements of the third component (ApJ 413, L129). Detailed studies of the O-type s-d systems AB Cru (A\&A 291, 185) and IU Aur (A\&A 284, 853), which contains a third component, have been made taking into account the effects of radiation pressure (A\&A 294, 723). A comparison of OB-type binaries, both detached and s-d, with models has also been done (A\&A 283, 144). Additional studies on well known s-d systems include $\beta$ Lyr and SV Cen (A\&A 291, 786), U Sge (A\&A 281, 433), and R CMa (ApJ 458, 371). Other studied s-d systems are V505 Sgr (AJ 106, 2051; 106, 2058; A\&AS 105, 47), with a third component, AQ Cas (AJ 108, 666), RZ Cas (A\&A 282, 821), RZ Dra (A\&A 285, 459), AT Peg (A\&A 285, 535), HU Tau (PASJ 46, 267; A\&A 297, 359), RZ Sct (AJ 108, 262), RT Per (Ann Shanghai Obs 15, 183), RS Cep (AJ 109, 345), VZ Psc (ApJ 455, 300) DN Ori (AJ 110, 1809) or UX Mon (AJ 110, 2385).

The chemical composition of Algol systems has been studied (MN 265, 581) confirming the carbon deficiencies in the primaries of eight systems. In detached binaries, lithium depletion in tidally locked binaries has been analyzed (ApJ 453, 819; ApJ 434, L71; A\&A 291, 507; A\&A 288, 829).

New apsidal motion studies of some EBs lead to improved ephemerides for DR Vul (MN 263, 527), CO Lac (A\&A 286, 875), DI Her (IBVS 4101, and 4161), AS Cam (A\&AS 116, 463), PV Cas (MN 277, 95; Ap\&SSci 204, 
191), Y Cyg (Obs 115, 188), V478 Cyg (PAZh 22, 149), V541 Cyg (IBVS 4217), and FT Ori (A\&AS 114, 143). Moreover, the apsidal motion rates of some of the systems with accurately determined stellar dimensions were analysed (EK Cep, GG Lup, AG Per, CO Lac, Y Cyg, V478 Cyg, AO Vel, PV Cas, and V539 Ara). A new list of eccentric eclipsing binaries was published (ExA 5, 91).

A global study of the available evidences of apsidal motion in the best known systems (A\&A 277, 487) shows that a reasonable agreement between theory and observations can now be reached if convective overshooting and an appropriate correction for stellar rotation is considered in the models. A revision of the ephemeris-curve equations for eclipsing binaries with apsidal motion (Ap\&SSci 226, 99) and a new discussion on the validity of the classical formula for tidal distortion has been published (ApJ 463, 284).

New linear and non-linear limb-darkening coefficients were published for UBV and uvby bands (AJ 106, 2096; A\&AS 110, 329), and for the RIJHK bands (A\&AS 114, 247). Gravity darkening has been studied in semidetached stars (PASJ 46, 613).

\section{BINARY STAR EVOLUTION}

\subsection{Origin and Formation (Alan P. Boss)}

Remarkable progress continues to be made in understanding the formation of binary stars, driven by both observational and theoretical advances. Comphreensive reviews were recently published (ARA\&A 32, 465; ARA\&A 33, 199; Rev. Mex. A\&Ap, Ser Conf 1, 165). Fragmentation appears to be close to being confirmed as the dominant formation mechanism.

High precision spectroscopic searches have begun to discover very low mass companions to nearby main sequence (MS) stars (Nat 378, 355; ApJ 464, L147; ApJ 464, L153). These companions appear to be brown dwarf stars or giant planets (Nat 379, 397); a cool brown dwarf companion to Gl 229 has been imaged (Science 270, 1478).

Surveys of companions to PMS stars continue to find a binary frequency at least as high as for nearby MS stars (ApJ 443, 625). For wide PMS binaries (120 AU or more), the binary frequency is about the same as for MS stars (A\&A 307, 121). The techniques employed for finding companions to PMS stars include infrared speckle imaging (AJ 106, 2005; A\&A 278, 129), optical CCD imaging (A\&A 278, 81), lunar occultations (A\&A 287,145 ), and spectroscopy (ARA\&A 32, 465). The implications of an excess of PMS binaries are unclear; the nearby MS binary frequency may have been underestimated, or binary stars may form at a higher rate in nearby star-forming regions. Higher binary frequencies in cold clouds have 
been predicted if fragmentation dominates (A\&A 286, 84). Regardless, the observations require that binary stars form prior to the PMS phase.

Most binary PMS stars appear to have formed at the same time (ApJ 427,961 ), consistent with a fragmentation origin. Masses of binary PMS stars can be found once orbits are determined (AJ 110, 753; A\&A 304, L17). Binary PMS stars often have disks (ApJ 429, L29) whose properties depend on the binary separation (ApJ 458, 312; A\&A 309, 493).

Binary protostellar objects are beginning to be discovered. Observational signatures of protostellar collapse are becoming more refined (ApJ 404, 232; ApJ 431, 767). The prototypical binary protostar is IRAS 16293 2422 (ApJ 402, 655). Another likely binary protostar lies in a single dense core inside the L1527 cloud (ApJ 463, L97). This object is so young that it has not completed a single orbit - it probably formed by fragmentation of its parent core. A hierarchical quadruple protostellar system has been found in the L1450 cloud (ApJ 452, L73). Analysis of young stellar object (YSO) clustering and binary separation data (MN 272, 213) revealed strong evidence for fragmentation on scales of dense cloud cores and smaller. Observations of pre-collapse cloud cores (MN 268, 276) show that these clouds have flat density profiles near their centers, similar to the Gaussian radial density profiles that are conducive to fragmentation (Rev Mex A\&Ap, Ser Conf 1,165$)$.

Forming binary stars by capture could occur for YSOs with large disks. However, studies of star-disk interactions imply that disk capture is still highly unlikely (ApJ 424, 292; ApJ 455, 252; but see MN 273, 1041).

Most theoretical work has concentrated on the fragmentation mechanism. (MN 283, 858) A study of cloud fragmentation with initial power-law density profiles confirms that fragmentation requires strong initial differential rotation. A modeled collapse of Gaussian profile clouds with an $\mathrm{m}=4$ density perturbation did not produce fragmentation (MN 268,625). Specific models concern the collapse of initially uniform density clouds with prolate shapes (MN 265, 905), the fragmentation in very thin, prolate clouds with differential rotation (ApJ 420,692), and the fragmentation in dense disks resulting from collisions between molecular cloud cores (MN 277, 705; MN $277,727)$.

A uniform density sphere was found to fragment into a binary linked by a thin bar which sub-fragmented into 9 more clumps (MN 294, 798). Following a binary formed in the standard isothermal test case to very late times, it was found that the binary survives (MN 277, 362). A standard nonisothermal test case has been published (ApJS 89, 345).

Fragmentation was also obtained in an adiabatic approximation model from clouds in the nonisothermal regime (MN 269, 837). Following Gaussian clouds into the nonisothermal regime with a radiative transfer code, it 
was found that differential rotation results in fragmentation even of clouds formally in virial equilibrium (MN 269, 837).

The results described so far refer to the first collapse phase. Adiabatic fragmentation was studied during the second collapse phase and, for the first time, obtained multiple long-lived fragments with separations on the order of 10 stellar radii, a possible explanation for forming CBS (MN 271, 999; MN 269, L45).

Taken altogether, this rapid observational and theoretical progress implies that fragmentation is the primary mechanism for forming binary stars. Further studies will serve to test and refine this bold statement.

\subsection{Binary System Dynamics and Magnetic Activity (Antonino F. Lanza and Marcello Rodonò)}

The orbital period variations which are observed in many CBS types (see $\S 3.2$, may assume an important role in the study of magnetically active stars. RS CVn-, W UMa-, Algol-type, as well as CV's show orbital period modulations with amplitudes of the order of $\frac{\Delta P}{P} \simeq 10^{-5}$ for RS CVns and Algols, with time scales from several decades up to possibly centuries, and $\frac{\Delta P}{P} \simeq 10^{-6}$ for CVs, with time scales from several years to a few decades.

Only a few mechanisms are able to explain alternate period changes on short time scales (A\&A 282, 775). Particularly attractive is the Applegate's mechanisms (ApJ 385, 621), that relates the orbital period modulation to the alternate changes of the gravitational quadrupole moment of one of the CBS components, due to more or less regular variations of its internal rotation regime along a magnetic solar-like cycle.

The best conditions for testing Applegate's hypothesis are provided by detached CBS without heavy mass exchanges or accrection processes, such as RS CVn systems. However, the proposed mechanism could be at work also in W UMa and Algol CBS with at least one component having a convective envelope, i.e., one of the necessary conditions for the development of magnetic activity (see ApJS 88, 199).

The predicted correlation between the $O-C$ curve and the mean brightness variation could not be properly tested with accurate and extended photometric data. Evidence in favour of the predicted correlation has been recently found for some RS CVn systems (A\&A 281, 811; A\&A 287, 817). However, the detailed analyses carried out in the latter papers show that the Applegate's mechanism can not account for the entire observed modulation, but only for short-term components ( $M N ~ 267,19)$. The simple phase relation by Applegate - based on simple energetic considerations - was not found in the case of the W UMa system AB And. 
A more stringent test has been performed searching for correlation between changes of the orbital period and rotation rate of the active component of RS CVn (A\&A 301, 75). A qualitative agreement with Applegate's hypothesis was found, though the rotation rate variation is only $20-30 \%$ of that predicted by the theory. However, this quantitative disagreement could be explained by a latitudinal differential rotation, or a peculiar evolution of the spots used as rotation tracers. However, other causes of orbital period modulation should not be excluded (see A\&A 291, 765; A\&A 293, 371).

Structural changes driven by magnetic dynamos could also take place in other classes of CBS such as CVs (PASP 106, 1075) and X-ray binaries and even in the companion of the millisecond pulsar PSR B1957+20 (ApJ 385, 621). However in those systems other physical mechanisms are certainly at work and do produce a very complex dynamical phenomenology (A\&A 279, L5). Moreover, the complex intrinsic variations observed in broad-band light curves should be taken into account to avoid spurious claims for orbital period changes (ApJ 438, 385; ApJ 459, 259).

\subsection{Mass Flow and Mass Exchange (Edward F. Guinan)}

This report covers the interval from July 1993 to July 1996. During this time hundreds of papers have been published that directly or indirectly relate to mass flow, mass exchange, accretion, and mass loss in CBS. New technologies and instruments as well as more sophisticated models and analysis techniques have permitted major advances to be made. It is obviously impossible to do justice to all of these studies and the great strides being made. Many papers on these topics are found in the conference proceedings and books quoted in $\S 1$ of this report.

Mass flows and mass exchange in CBS are inevitable consequences of the component stars being near enough to interact physically in such a way as to alter significantly their evolution from that of corresponding single stars (see Obs 114, 212). The major episodes of mass flow, exchange and mass loss occur in s-d systems when the more massive star evolves off the main-sequence and fills its Roche lobe. Rapid mass exchange and loss can also take place during the final stages of binary star evolution when one of the components is a degenerate star and the former secondary star expands (or the orbit shrinks from AML) so that it approaches its Roche lobe and mass transfer/loss insues. These systems include CVs, X-ray binaries, and some symbiotic stars. However, it is now generally appreciated that mass flows and mass exchange also take place in detached systems that contain stars with strong stellar winds, such as hot, massive O-B and WR stars, supergiants (the $\zeta$ Aur systems) and M-giants (the symbiotics). It is now recognized that mass flows also can result from magnetic activity 
(flares, prominences, magnetically driven winds etc.) of chromosphericallyactive binaries (the RS CVn stars) and from rapid rotation of one of the components. Also important are mass flows that include winds, jets, and bipolar flows originating from the accretion disks.

Important advances in the theory and modeling of gas flows have come from the application of hyrodynamic (HD) simulations to mass transfer in close binaries, e.g., 2-D HD simulations of mass transfer for short period Algols (AJ 445, 939) and for binaries that do not fill their Roche lobes (AZh $71,560)$. These simulations produce asymmetric accretion structures with many features seen in $\mathrm{H} \alpha$ spectroscopy and show that that the physical properties of the stellar wind substantially influence the structure of the gas flow. Evolutionary models for non-conservative mass exchange have been computed for some specific sytems such as: U Sge (A\&A 281, 433), $\beta$ Lyr and SV Cen (A\&A 291, 786) and for AM Her stars (MN 266, L1). Also, more physically realistic accretion disk models have been developed and applied (e.g., A\&A 289, 411; ApJ 434, 738; ApJ 426, 294).

Major advances have been made from the direct imaging, or indirectly by using Doppler tomography or eclipse mapping techniques, of the gas flows and accretion structures themselves. For example, the inner region of the symbiotic system R Aqr was directly imaged with the HST/FOC (A\&A $287,154)$. These observations favor the wind collision and nozzle theories for the origin of the jet in R Aqr. Also with the HST/FOC, UV images of a bipolar nebula for another symbiotic HM Sgr, similar to that in R Aqr, were obtained (PASP 105, 1273). The binary SS433 also contains jets and, by CCD X-ray spectrometers on ASCA, the Doppler shifted X-ray emission lines of Fe XXV and Fe XXVI from the jet have been resolved (PASJ 46, L147). This is the first direct detection of two Doppler shifted beams in the X-ray spectrum of SS433. In a recent study of $\beta$ Lyr (A\&A 312, 879), evidence of polar jets is found from the analysis of extensive interferometric, spectroscopic and photometric observations. The bulk of the $\mathrm{H} \alpha$ and $\mathrm{HeI}$ 6678 emission appears to arise from jets of gas associated with the impact flow region of the accretion disk.

Doppler tomography and eclipse mapping techniques are providing unprecendented information on the properties and effects of gas flows in CBS, e.g., the spectral eclipse maps of the accretion disk in the nova-like variable UX UMa (A\&A 283, 441), the Doppler tomography of the polar VV Pup (A\&A 283, 508) in which the gas stream is detected and the absence of an accretion disk is confirmed, and the eclipse mapping of the accreting magnetic WD in the AM Her system DP Leo using HST time resolved UV spectroscopy (ApJ 430, 323). Although much of the work on Doppler tomography has been applied to CVs and AM Her systems, recently tomographic methods have been applied to short-period Algols (e.g., ApJ 438, 
L106; ApJ 459, 249; ApJ 459, L99), yielding detailed maps of the gas flows and the properties of the accretion structures.

Much work has also been done on wind flows and accretion processes in $\zeta$ Aur systems and symbiotics. These include modeling of winds and accretion in the $\zeta$ Aur systems AL Vel (AJ 107, 729), $\zeta$ Aur (ApJ 466, 979), and 32 Cyg (A\&A 291, 535), and the symbiotic systems CH Cyg (A\&A 287, 543), MWC 560 (AJ 108, 671), and SY Mus (A\&A 288, 819). Also intriguing are the studies of wind flows and clashing winds in CBS with OB or WR components: V444 Cyg (Adv Sp Res 13, 295; ApJ 422, 810). A method for determining the colliding wind parameters in (WR+OB) interacting system from their X-ray spectrum is presented in $A Z h ~ 71,78$. In a related study (Obs 114, 1123), the winds of the O-B stars of the eclipsing binary Y Cyg are studied from IUE spectra.

Traditional ground-based photometry and spectroscopy (cf $\S 12,3.3$, and 3.4) continue to produce important results on mass flows and mass exchange in CBS. For example, the OI 7774 absorption line has been used as a tracer of streams and disks in Algol systems (AJ 109, 1269). Also, studies of period changes provide indirect but important clues on mass exchange and mass loss in CBS.

A note in passing is the termination of the operation of the IUE satellite on September 1996. Over the last 19 years this satellite has contributed in a major way to our understanding of gas flows, mass exchange and accretion in CBS. Major advances are expected in the near future when several optical interferometers come on line that are capable of directly imaging gas streams and associated phenomena. Also, with the upcoming launches of two powerful X-ray satellites, AXAF and XMM, X-ray spectroscopy and tomography of bright $\mathrm{X}$-ray binaries will be feasible. Exciting times are ahead for close binary stars!

\subsection{Neutron Star X-ray Binaries (Luigi Stella)}

The entire subject has been reviewed in the excellent monograph $X$-ray Binaries (Cambridge Univ Press), which reflects the development of the field through early 1994.

Considerable progress has been achieved in the study of pulsating X-ray binaries. About ten new X-ray pulsars have been discovered (some of which are transients). Several of these are ROSAT serendipitous sources in the Magellanic Clouds (MCs). RX J0059.2-7138, a $2.7 \mathrm{~s}$ transient X-ray pulsar in the LMC, is especially interesting in that it displayed a pronounced very soft X-ray component consistent with the spectral shape and luminosity of super soft sources (ApJ 427, L25), which likely host white dwarfs accreting at a very high rate. 
Following the discovery of $9 \mathrm{~s}$ pulsation from the ultrasoft source $4 \mathrm{U} 0142$ +614 (ApJ 433, L25), a new group of 4-5 anomalous accreting pulsars with 5-9 s spin periods and unusually soft X-ray spectra (resembling the ultrasoft spectra of accreting black hole candidates in their high state, rather than the hard spectra of most X-ray pulsars) has been identified (ApJ 442, L17). This group is also characterized by low X-ray luminosities $\left(10^{35}-10^{36} \mathrm{erg}\right.$ $\mathrm{s}^{-1}$ ) and secular spin-down, indicating that the neutron stars are close to their equilibrium period and have relatively low magnetic fields of few $\times 10^{11} \mathrm{G}$. These X-ray pulsars either have a very low mass companion, or are isolated and accrete from a residual disk, possibly following a ThorneZytkov evolutionary phase (A\&A 299, L41; ApJ 463, L83).

Medium resolution X-ray spectroscopy with ASCA revealed a pronounced Ne K-line complex around $1 \mathrm{keV}$ in the spectrum of the 4U1626647 , testifying that its low mass companion has undergone a He burning phase (ApJ 449, L41). The variation of the Fe K-shell line complex of Cen $\mathrm{X}-3$ over an eclipse provided important clues on the geometry and physical state of the matter producing the different line components (PASJ 48, 425). CGRO observations of the transient X-ray pulsar A0535+26 confirmed the presence of a cyclotron resonant scattering feature at $110 \mathrm{keV}$, corresponding to the strongest magnetic field $\left(\sim 10^{13} \mathrm{G}\right)$ yet measured in an accreting neutron star (ApJ 438, L25); moreover the frequency dependence of the 30$70 \mathrm{mHz}$ quasi periodic oscillations (QPOs) on source flux allowed to test the predictions of the beat frequency model to an unprecedented accuracy (ApJ 459, 288).

Among transient systems, GRO 1744-28, a $0.5 \mathrm{~s}$ X-ray pulsar in a $12 \mathrm{~d}$ orbit around a low mass evolved companion, displayed an extraordinary variety of intense X-ray bursts, which repetead on timescales from minutes to hours (Nat 379, 799; Nat 381, 291). These bursts are believed to originate from a gravitational instability and are reminiscent of those from the Rapid Burster (ApJ 462, L39) and SMC X-1 (ApJ 371, 332).

The short term X-ray variability of old accreting neutron stars in low mass X-ray binaries (LMXRBs) is being investigated to an unprecedented detail by the large effective area detectors on board RXTE. Especially interesting is the discovery of very high frequency QPOs $(500-1000 \mathrm{~Hz})$ in several LMXRBs, including Sco X-1. QPOs at two different and variable frequencies are occasionally observed.

An interesting result is the transient hard X-ray emission up to energies of $>100 \mathrm{keV}$ that has been discovered in a number of LMXRBs containing an old neutron star (ApJS 92, 505). Such a hard component was previously considered a unique spectral feature of accreting black hole candidates. 


\subsection{Binary-related Eruptive Phenomena, Accretion Disks and Flares (Paula Szkody and Craig Wheeler)}

The past 3 years have witnessed the start of a new era of immediate and detailed knowledge of eruptive phenomena through electronic mail alerts (the vsnet group in Japan, the AAVSO and the nova exploder at nova@nova.la.asu.edu in the US) and the wide availability of charts and light curves of ongoing eruptions through Web pages (http://www.kusastro. kyoto-u.ac.jp/vsnet/ and http://www.aavso.org). These alerts and datasets have provided a multitude of new information on previously little-studied systems, especially for identifying new SU UMa stars by their superhump periods during outbursts. Relatively rare eruptions of the dwarf nova AL Com occurred in April 95 and the old nova GK Per in Feb 96 as well as the new novae N Cas 93 = V705 Cas (slow with dust), N Sgr 94 (symbiotic), $\mathrm{N}$ Oph 94, N Cir 95, N Cas 95 (very slow, with multiple brightenings), $\mathrm{N}$ Cen $95, \mathrm{~N}$ Aql 95 and N Sgr 96.

Major reviews of the entire field have been published in summary books and conference proceedings (cf. $\S 1$ ). Reviews on the specific topics of DQ Her stars (PASP 106, 209), dwarf novae outbursts (PASP 108, 39), superoutbursts (Ap\&SSci 226, 187) and novae (in Phys Proc in Astrophys., Springer-Verlag) have appeared.

Long term (years) photometric monitoring of close to 100 old novae and novalike systems with the Indiana Roboscope telescope resulted in the discovery of several different modes of lightcurve behavior (Ap\&SSci 205, 75 ), including the RZ LMi (or ER UMa) stars, a class of SU UMa stars with supercycles from 18-45 days (PASJ 47, 163; PASP 107, 443), old novae with sine-like variations on timescales of about a month, and old novae with periodic outbursts.

Doppler tomography continued to play a major role as a technique to study the characteristics of the accretion disk and stream in disk systems and the stream flow in magnetic systems: emission line tomograms for 18 CVs (ApJS 93, 519), stream flows in SW Sex stars (ApJ 431, L107), streams outflowing from the system rather than accreting to a disk on the DQ Her star AE Aqr (ASP Conf Ser 85, 196), accretion flow from the secondary to the magnetic white dwarf on the AM Her star HU Aqr (ASP Conf Ser 85, 166). Significant progress has been made in understanding the early ultraviolet and optical spectra of novae using a spherical, expanding, model stellar atmosphere computer code developed by Hauschildt and his collaborators (ApJ 393，307; JQSRT 47，433; JQSRT 50, 301; ApJ 447, 829; JQSRT $54,987)$. They use an Accelerated Lambda-iteration method to solve the time independent, spherically symmetric, fully relativisitic radiative transfer equation for lines and continua. They also solve, simultaneously, the 
multi-level, non-LTE rate equations for 15 elements (3742 NLTE levels) using a rate-operator splitting method. These methods have been applied to V1974 Cyg (ApJ 422, 831), SN 1993J (ApJ 426, 334; ApJ 441, 170), V705 Cas 1993 (AJ 108, 1008), SN 1994i (MN 279, 170). An important improvement has come through the addition of Fe II in non-LTE to the code (ApJ 462, 386), significantly improving the agreement of the calculations with the observations.

The ejecta abundances for a number of novae have been published: Nova V1974 Cyg (AJ 111, 869), V351 Pup (MN 279, 280), and 10 novae (A\&A $291,869)$.

Spacecraft data have greatly contributed to the understanding of CBS phenomena. Time resolved UV spectra obtained with HST were used to separate the white dwarf, accretion disk and bright spot of the dwarf nova OY Car (ApJ 426, 294). The theoretically expected boundary layer was lacking in EUVE observations of SS Cyg (ApJ 446, 842), but has been very recently detected during an outburst of $U$ Gem. The possibility that the missing boundary layer could be due to rapid rotation of the white dwarf was ruled out from high resolution HST studies of U Gem and VW Hyi (ApJ 430, L53; ApJ 445, L31).

The heating and cooling of the white dwarf following outbursts in dwarf novae and changing accretion states in magnetic systems was studied by several groups with HST, IUE and HUT (ApJ 424, L49; ApJ 109, 1746; ApJ 444, L97; A\&A 303, 127; ApJ 460, 939). EUVE and ASCA data showed that the spectra of magnetic systems were sometimes compatible with irradiation from the accretion column (ASP Conf Ser 85, 132), but at other times, with deep heating by blobs (ApJ 445, 921). This deep heating is postulated to account for the soft X-ray excess that exists in many magnetics (MN 270, 692).

The search for counterparts to the sources discovered in the ROSAT allsky-survey led to the identification of a large number of new CVs, especially magnetic systems (ASP Conf Ser 85, 99): 3 systems were found in NGC 6397 (ApJ 455, L47) and other detections have been announced. Most of the luminous supersoft sources (Ap\&SSci 205, 409) were explained with models of steady hydrogen burning on accreting white dwarfs in binaries (ApJ 421, 261 , which included novae after outburst and symbiotic stars. The turnoff of the nuclear burning phase was witnessed in 2 novae: GQ Mus 10 yrs after its outburst (ApJ 438, L95) and V1974 Cyg 1.5 yrs after outburst (ApJ 456, 788). V1974 Cyg was the first classical nova to be observed from gamma ray through radio, which provided a complete picture of the physical conditions throughout the outburst of a neon nova (AJ 111, 869).

ASCA observations of photoionized lines have led to deeper understanding of the environments of binary X-ray sources. RXTE has very recently 
resolved the first millisecond and submillisecond QPOs in X-ray binaries, thus providing constraints on theories of radiation in the vicinity of neutron stars. CGRO BATSE observed comparable long term spin down and spin up of accreting pulsars, in contradiction to popular theories. CGRO BATSE has also verified the correspondence of torque and spin acceleration in transient pulsars. RXTE sees oscillations with periods less than 1 $\mathrm{ms}$ in low magnetic field accreting pulsars and has given evidence that the neutron star in at least 1 burster has a rotation period of $3 \mathrm{~ms}$.

One of the most suprising recent developments is due to CGRO BATSE: many of the soft X-ray transients are very likely to be black holes with rather small mass companions. There are now six such systems with welldefined mass functions in excess of 3 solar masses. The exponential decline observed in many of these systems puts strong constraints on the nature of the disk viscosity and points strongly to an origin in a dynamo driven by internal waves (ApJ 435, 389; ApJ 454, 880). These objects have a hard Xray/gamma-ray components that is very reminiscent of QSO's and AGN's, and two of them have been observed to have superluminal radio jets. Some of them have revealed red shifted annihilation radiation, but an alternative explanation involves de-excitation gamma rays from spallation to form lithium. The companions of some of these systems (and some neutron star systems) show lithium enhancements. GINGA, EXOSAT, and now RXTE have found quasi-periodic oscillations at a various frequencies and their first harmonic from black hole transients. Many of these frequencies are similar to neutron star sources, suggesting a common origin.

Recent observations have confirmed that Type Ia SN are not standard candles, but have a true dispersion in maximum brightness that correlates with the rate of decline. We still do not know if Type Ia supernovae arise in some form of cataclysmic variable, in double degenerate systems, or some other configurations. The supersoft X-ray sources may be related to the progenitors of Type Ia supernovae. The physics of the thermonuclear explosions underlying Type Ia supernovae has also advanced in a major way, with the insight that multi-dimensional effects are paramount. Current searches are routinely discovering Type la events at a redshift of about 0.5 , while they have been observed to a red shift as large as 0.8 .

Acknowledgments. I should like to express my warmest thanks to the distinguished contributors to this report for their serious and competent effort in offering a valuable service to Commission 42 members and to the astronomical community at large.

Marcello Rodonò 